\title{
Production Rate of Second KK Gauge Bosons in UED Models at LHC
}

\author{
Shigeki Matsumoto \\ Department of Physics, University of Toyama, \\ Toyama 930-8555, Japan \\ E-mail: smatsu@sci.u-toyama.ac.jp \\ Joe Sato* $^{*}$ and Masato Yamanaka ${ }^{\dagger}$ \\ Department of Physics, Saitama University, \\ Shimo-okubo, Sakura-ku, Saitama, 338-8570, Japan \\ *E-mail: joe@phy.saitama-u.ac.jp \\ $\dagger$ E-mail: masa@krishna.phy.saitama-u.ac.jp \\ Masato Senami \\ Department of Micro Engineering, Kyoto University, \\ Kyoto 606-8501, Japan \\ E-mail: senami@me.kyoto-u.ac.jp
}

\begin{abstract}
We calculate the production rates of the second Kaluza-Klein (KK) photon $\gamma^{(2)}$ and Z boson $Z^{(2)}$ at the LHC including all significant processes in the minimal universal extra dimension (MUED) model. For discrimination of the MUED model from other TeV scale models in hadron collider experiments, $\gamma^{(2)}$ and $Z^{(2)}$ play a crucial role. In order to discuss the discrimination and calculate their production rates, we derive effective Lagrangian containing KK number violating operators. We show that KK number violating processes are extremely important for the compactification scale larger than $800 \mathrm{GeV}$. We find that, with an integrated luminosity of $100 \mathrm{fb}^{-1}, \gamma^{(2)}$ and $Z^{(2)}$ are produced $10^{6}$ - $10^{2}$ for the compactification scale between $400 \mathrm{GeV}$ and $2000 \mathrm{GeV}$.
\end{abstract}

Keywords: universal extra dimension model, LHC, second KK gauge bosons

\section{Introduction}

Universal Extra Dimension (UED) models ${ }^{1}$ are one of attractive candidates for new physics at TeV scale. Among various UED models, the simplest and the most popular one is called the Minimal UED (MUED) model. The MUED model is defined on the five dimensional space-time, where the extra dimension is compactified on an $S^{1} / Z_{2}$ orbifold. In the MUED model, only two parameters are newly introduced to the SM. One is the compactification scale of the extra dimension $1 / R$, the inverse of the radius of $S^{1}$ circle, and the other is the cutoff scale of the MUED model $\Lambda$. In this article, we take $\Lambda$ to be $\Lambda R=20$. In the MUED model, all SM fields can propagate into compactified extra dimension, and fields with a fifth dimensional momentum 
$n / R$ behave as new heavy particles with a mass $m_{n}=\sqrt{(n / R)^{2}+m_{S M}^{2}+\delta m_{n}^{2}}$ from the viewpoint of four dimensional field theory. Here analytical expression of the radiative corrections $\delta m_{n}$ are given in Ref. 2. These new particles are called Kaluza-Klein (KK) particles, $n$ is called the KK number $(n=0$ for SM particles, $n=1,2, \ldots$ for $\mathrm{KK}$ particles), and $m_{S M}$ represents the mass of the corresponding SM particle. They can give plausible explanations for the existence of dark matter, ${ }^{3}$ SM neutrino masses which are embedded in extended models, ${ }^{4}$ and so on.

Since the translational invariance along the extra dimension direction is broken due to the orbifolding, the fifth dimensional momentum (KK number) is no longer conserved. Nevertheless, the subgroup of the translational invariance remains unbroken, which is called the KK parity. Under the parity, particles with even (odd) KK number have plus (minus) sign, and the product of the sign is conserved in each process. Because of the KK parity, the lightest KK particle (LKP) is stable and provided as a candidate for dark matter. The relic abundance of the KK particle dark matter has been calculated, ${ }^{5}$ and it turns out that $1 / R$ should be in the range of $500 \mathrm{GeV}-1500 \mathrm{GeV}$. The MUED model, therefore, would be explored at the LHC.

The confirmation of the MUED model needs the discovery of KK particles. Though the LHC can produce KK particles, it is difficult to confirm that they are indeed the KK particles, because new particles predicted in various $\mathrm{TeV}$ scale models give quite similar signatures to each other. Therefore it is very important to understand how they can be identified as the KK particles. In this article, we discuss the discrimination of the MUED model from other TeV scale models.

An idea to discriminate the MUED model from other models has been proposed. ${ }^{6}$ The essence of the discrimination is the discovery of the second KK particles. The discovery of the second KK particles suggests the existence of the MUED model, since their masses are peculiar which is almost equal to $2 / R$ and $1 / R$ is expected by the masses of the "first KK" particles. In particular, the second KK photon $\gamma^{(2)}$ and $\mathrm{Z}$ boson $Z^{(2)}$ play an important role for the search of the second KK particles. They are able to decay into two charged leptons through the KK number violation operators. It is possible to reconstruct their masses clearly from the charged dileptons. Connecting their masses and those of the first KK particles, we can confirm the realization of the MUED model. Hence, $\gamma^{(2)}$ and $Z^{(2)}$ are the key ingredients for the discrimination. We therefore calculate their production rates at the LHC. This proceedings is based on the work. ${ }^{7}$

\section{Productions of $\gamma^{(2)}$ and $Z^{(2)}$}

In this section, we discuss the Lagrangian relevant to the productions of $\gamma^{(2)}$ and

$Z^{(2)}$ bosons. With the Lagrangian we calculate the branching ratios of $g^{(2)}$ and $q^{(2)}$, which are necessary for the discussion of the indirect production of $\gamma^{(2)}$ and $Z^{(2)}$.

\subsection{Lagrangian for $\gamma^{(2)}$ and $Z^{(2)}$ productions}

Firstly, we show the Lagrangian conserving the KK number relevant to gauge bosons, 


$$
\begin{aligned}
\mathcal{L}_{\text {con }}=- & g_{i} \sum_{n=1}^{\infty}\left[\bar{f}^{(n)} t^{a} \gamma^{\mu} f^{(n)} V_{i \mu}^{(0) a}\right. \\
& \left.+\bar{f}^{(n)} t^{a} \gamma^{\mu} P_{L(R)} f^{(0)} V_{i \mu}^{(n) a}+\bar{f}^{(0)} t^{a} \gamma^{\mu} P_{L(R)} f^{(n)} V_{i \mu}^{(n) a}\right] \\
- & \frac{g_{i}}{\sqrt{2}} \sum_{n, m=1}^{\infty}\left[\bar{f}^{(n)} t^{a} \gamma^{\mu} \gamma^{5} f^{(m)} V_{i \mu}^{(n+m) a}\right. \\
& \left.+\bar{f}^{(n+m)} t^{a} \gamma^{\mu} f^{(n)} V_{i \mu}^{(m) a}+\bar{f}^{(n)} t^{a} \gamma^{\mu} f^{(n+m)} V_{i \mu}^{(m) a}\right] \\
+ & g_{i} f_{i}^{a b c} \sum_{n=1}^{\infty}\left[\left(\partial_{\mu} V_{i \nu}^{(0) a}\right) V_{i}^{(n) b \mu} V_{i}^{(n) c \nu}\right. \\
& \left.+\left(\partial_{\mu} V_{i \nu}^{(n) a}\right) V_{i}^{(n) b \mu} V_{i}^{(0) c \nu}+\left(\partial_{\mu} V_{i \nu}^{(n) a}\right) V_{i}^{(0) b \mu} V_{i}^{(n) c \nu}\right]
\end{aligned}
$$

where the summation over $i, a, b, c$ are implicitly made. $f^{(n)}, g_{i}, t^{a}, V_{i \mu}^{a}$ are listed in Table 1. In the third part of the Lagrangian, $f_{i}^{a b c}$ is the structure constant of $S U(3)$ for gluon and that of $S U(2)$ for W boson. As long as we use the KK number conserving Lagrangian, second KK particles are produced in pair due to the KK number conservation, and hence the production rates are suppressed due to their small phase spaces.

Table 1. $f^{(n)}, g_{i}, t^{a}, V_{i \mu}^{a}$ in the KK number conserving Lagrangian. $B, W, g$ are $U(1), S U(2), S U(3)$ gauge bosons, $g^{\prime}, g_{2}, g_{s}$ are $U(1), S U(2), S U(3)$ gauge coupling constants, and $\sigma^{a}, \lambda^{a}$ are Pauli matrices, Gell-Mann matrices respectively.

\begin{tabular}{lccc}
\hline n-th KK fermion $f^{(n)}$ & $g_{i}$ & $t^{a}$ & $V_{i \mu}^{a}$ \\
\hline$S U(2)$-singlet charged lepton $E^{(n)}$ & $-g^{\prime}$ & 1 & $B_{\mu}$ \\
\hline$S U(2)$-doublet lepton $L^{(n)}$ & $-(1 / 2) g^{\prime}$ & 1 & $B_{\mu}$ \\
& $g_{2}$ & $\sigma^{a} / 2$ & $W_{\mu}^{a}$ \\
\hline$S U(2)$-singlet up-type quark $U^{(n)}$ & $(2 / 3) g^{\prime}$ & 1 & $B_{\mu}$ \\
& $g_{s}$ & $\lambda^{a} / 2$ & $g_{\mu}^{a}$ \\
\hline$S U(2)$-singlet down-type quark $D^{(n)}$ & $-(1 / 3) g^{\prime}$ & 1 & $B_{\mu}$ \\
& $g_{s}$ & $\lambda^{a} / 2$ & $g_{\mu}^{a}$ \\
\hline$S U(2)$-doublet quark $Q^{(n)}$ & $(1 / 6) g^{\prime}$ & 1 & $B_{\mu}$ \\
& $g_{2}$ & $\sigma^{a} / 2$ & $W_{\mu}^{a}$ \\
& $g_{s}$ & $\lambda^{a} / 2$ & $g_{\mu}^{a}$ \\
\hline
\end{tabular}

Next we discuss the KK number violating interactions. The effective Lagrangian for the KK number violating operators turns out to be :

$\mathcal{L}_{\text {vio }}=\frac{x_{i}}{4}\left\{N_{i}(f) c_{t}+\left[9 C_{j}(f)-\frac{23}{3} C_{j}(G) \delta_{i j}+\frac{n_{j}}{3} \delta_{i j}\right] c_{j}\right\} \bar{f}^{(0)} t_{i}^{a} \gamma^{\mu} P_{L(R)} f^{(0)} V_{i \mu}^{(2) a}$, 


$$
c_{j} \equiv \frac{\sqrt{2} x_{j}^{2}}{16 \pi^{2}} \log \frac{\Lambda^{2}}{\mu^{2}}, \quad c_{t} \equiv \frac{\sqrt{2} y_{t}^{2}}{16 \pi^{2}} \log \frac{\Lambda^{2}}{\mu^{2}} .
$$

Here $y_{t}$ is the top Yukawa coupling constant, $x_{i}, n_{i}, C_{i}(f), C_{i}(G), t_{i}^{a}$ are listed in Table 2, and $N_{i}(f)$ is listed in Table 3. Indices $i, j$ run over the SM gauge interactions $U(1), S U(2)$, and $S U(3)$, and summation over $f$ is implicitly made. The renormalization scale is denoted by $\mu$.

Table 2. Coefficient in the KK number violating operator in the effective Lagrangian (Eq. (2)). $g^{\prime}, g_{2}, g_{s}$ are $U(1), S U(2), S U(3)$ gauge coupling constants, and $Y_{f}$ is $U(1)$ hypercharge. $\sigma^{a}, \lambda^{a}$ are Pauli and Gell-Mann matrices, respectively.

\begin{tabular}{cccc}
\hline & $U(1)$ & $S U(2)$ & $S U(3)$ \\
\hline$x_{i}$ & $g^{\prime} Y_{f}$ & $g_{2}$ & $g_{s}$ \\
$n_{i}$ & 1 & 2 & 0 \\
$C_{j}(f)$ & $Y_{f}^{2}$ & $3 / 4$ & $4 / 3$ \\
$C_{i}(G)$ & 0 & 2 & 3 \\
$t_{i}^{a}$ & $\mathbf{1}$ & $\sigma^{a} / 2$ & $\lambda^{a} / 2$ \\
\hline
\end{tabular}

Table 3. Coefficient $N(f)$ in the KK number violating operator in the effective Lagrangian (Eq. (2) ). $Q_{3}$ is the third generation $S U(2)$-doublet quark, and $T$ is $S U(2)$ singlet top quark.

\begin{tabular}{ccccccc}
\hline & $Q_{3}^{(0)} Q_{3}^{(0)} \gamma^{(2)}$ & $T^{(0)} T^{(0)} \gamma^{(2)}$ & $Q_{3}^{(0)} Q_{3}^{(0)} W^{(2)}$ & $Q_{3}^{(0)} Q_{3}^{(0)} g^{(2)}$ & $T^{(0)} T^{(0)} g^{(2)}$ & other \\
\hline$N(f)$ & 1 & 5 & 1 & 1 & 1 & 0 \\
\hline
\end{tabular}

\subsection{Production processes of $\gamma^{(2)}$ and $Z^{(2)}$}

We are now in a position to discuss the production processes of $\gamma^{(2)}$ and $Z^{(2)}$. At the LHC, $\gamma^{(2)}$ and $Z^{(2)}$ are produced through two type of processes : (1) direct productions and (2) indirect productions via the cascade decays of the second KK colored particles. The production cross sections of $\gamma^{(2)}$ and $Z^{(2)}$ has originally been calculated in Ref. 6, and their calculation includes all of the KK number conserving processes and the direct 1-body production processes of the second KK gauge bosons. In this article, we calculate the production cross sections of $\gamma^{(2)}$ and $Z^{(2)}$ including all significant processes. For example, our calculation includes $p p \rightarrow q^{(2)} q^{(0)}$, $p p \rightarrow \gamma^{(2)} q^{(0)}, p p \rightarrow q^{(2)} \bar{q}^{(0)}$ and so on. Importantly, these processes provide large contributions to $\gamma^{(2)}$ and $Z^{(2)}$ productions, particularly for large $1 / R(\gtrsim 800 \mathrm{GeV})$. We show the relevant processes to the $\gamma^{(2)}$ production through KK number violating interactions in Figure 1 - 3. We skip the discussion of the $\gamma^{(2)}$ production through KK number conserving interactions. The processes of $Z^{(2)}$ production are almost same as the $\gamma^{(2)}$ production, so that we can skip the discussion of the $Z^{(2)}$ production.

\section{Numerical results}

In Figure 4 (Figure [5), we show the production cross section of $\gamma^{(2)}\left(Z^{(2)}\right)$ as a function of $1 / R$. In the calculation, we have used the CTEQ6L code $^{8}$ as a parton 

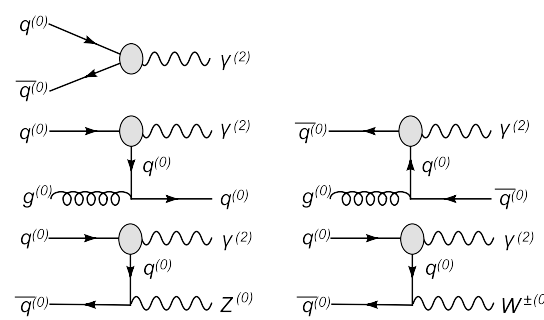

Fig. 1. The direct production of $\gamma^{(2)}$ through KK number violating processes. The colored circle stands for the KK number violating vertex.
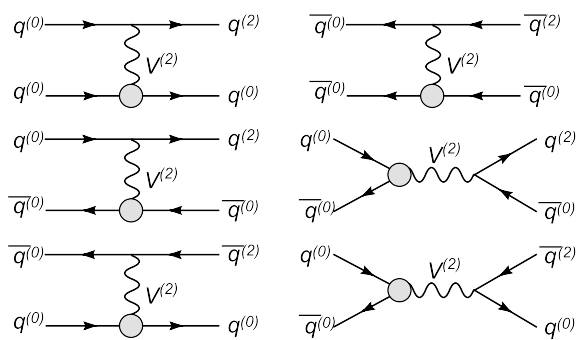

Fig. 2. The production of $q^{(2)}$ through KK number violating processes. $V^{(2)}$ stands for $\gamma^{(2)}$, $W^{ \pm(2)}, Z^{(2)}$, and $g^{(2)}$. The colored circle stands for the KK number violating vertex.
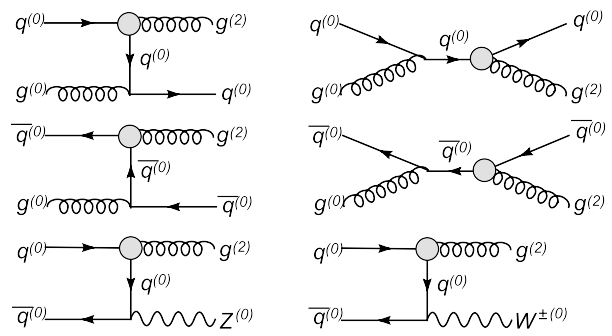

Fig. 3. The production of $g^{(2)}$ through KK number violating processes. The colored circle stands for the KK number violating vertex.

distribution function, the calculations of the cross sections have been performed by using the calcHEP ${ }^{9}$ implementing the Lagrangian Eq. (2) derived in the previous section. Red solid line shows the total cross section of $\gamma^{(2)}\left(Z^{(2)}\right)$ production.

Assuming an integrated luminosity of $100 \mathrm{fb}^{-1}, \gamma^{(2)}$ and $Z^{(2)}$ will be produced $10^{6}-10^{2}$ for $400 \mathrm{GeV} \leq 1 / R \leq 2000 \mathrm{GeV}$. Once $\gamma^{(2)}$ and $Z^{(2)}$ are produced, they decay into dileptons with about $1 \%$ branching ratios. In Table 4 , we show the number of the dilepton signals with assuming the luminosity $100 \mathrm{fb}^{-1}$. For $1 / R \lesssim$ $1600 \mathrm{GeV}$, dilepton signals will be observed at least 1 event. If the MUED model is realized by the nature, in addition to the dilepton signals from the decay of $\gamma^{(2)}$ and $Z^{(2)}$, many new particles with degenerate mass spectrum around $1 / R$, i.e., the first KK particles are discovered. Connecting the observational results of dilepton 


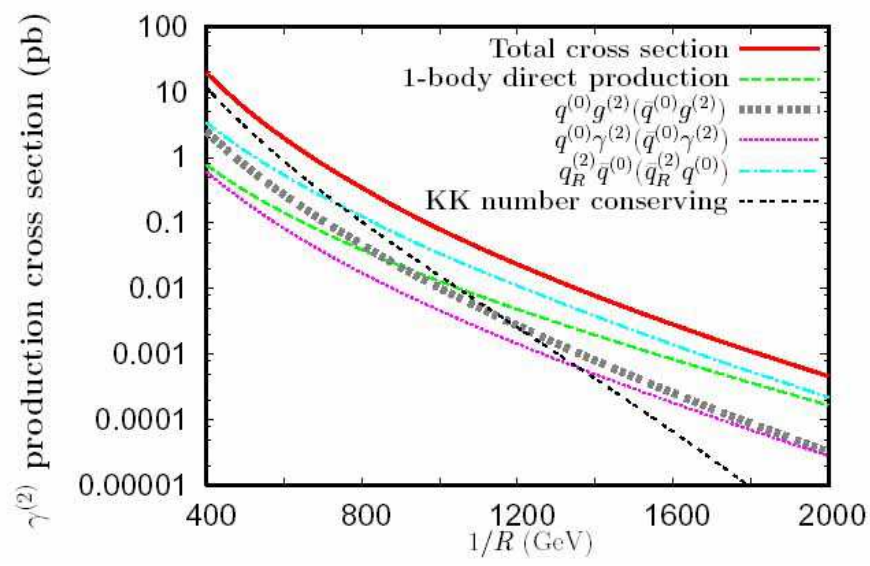

Fig. 4. The production cross sections of $\gamma^{(2)}$. Red solid line shows the total cross section, and each line shows partial cross section for each process as denoted in the legend.

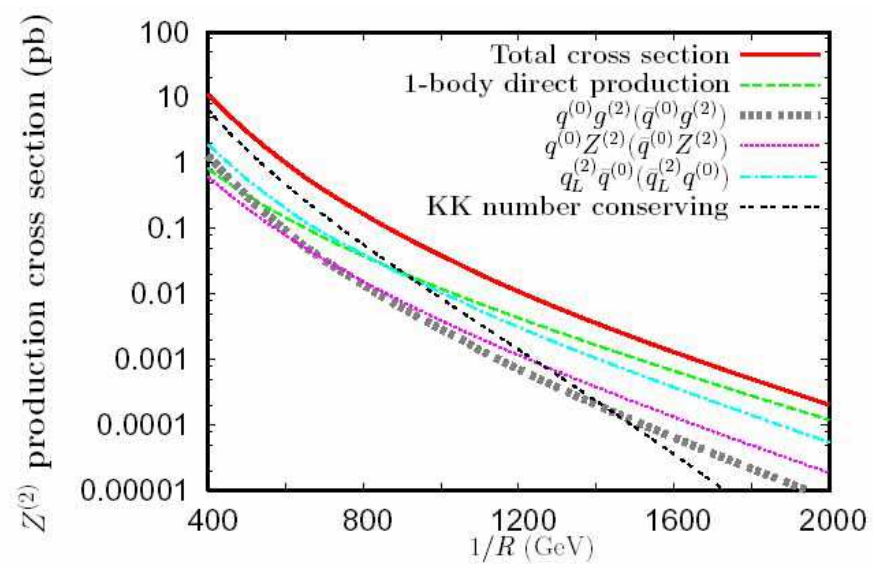

Fig. 5. The production cross sections of $Z^{(2)}$. Red solid line shows the total cross section, and each line shows partial cross section for each process as denoted in the legend.

signals and the discovery of the first KK particles, it is possible to confirm the MUED model.

Table 4. The number of the dilepton signals at the LHC with $100 \mathrm{fb}^{-1}$.

\begin{tabular}{ccc}
\hline $1 / R$ & dileptons from $\gamma^{(2)}$ & dileptons from $Z^{(2)}$ \\
\hline $400 \mathrm{GeV}$ & $1.5 \times 10^{4}$ & $9.4 \times 10^{3}$ \\
$800 \mathrm{GeV}$ & $2.9 \times 10^{2}$ & $1.6 \times 10^{2}$ \\
$1200 \mathrm{GeV}$ & $2.1 \times 10$ & $1.2 \times 10$ \\
$1600 \mathrm{GeV}$ & 2.6 & 1.4 \\
$2000 \mathrm{GeV}$ & $4.4 \times 10^{-1}$ & $2.3 \times 10^{-1}$ \\
\hline
\end{tabular}




\section{Summary}

At the LHC, the discrimination of the MUED model from other models is difficult, because the signals of new particles of $\mathrm{TeV}$ scale models are quite similar to each other. For the discrimination, we focused on the existence of the KK tower. Once $\gamma^{(2)}$ and $Z^{(2)}$ are produced, they can decay into dilepton which provides a very clean signal of the second KK particles. The discovery of the second KK particles, indicates the existence of the KK tower and hence will lead to the confirmation of the MUED model. In order to estimate the dilepton events from $\gamma^{(2)}$ and $Z^{(2)}$, we have calculated the production rates of $\gamma^{(2)}$ and $Z^{(2)}$ at the LHC.

We have calculated the KK number violating operators. They play a crucial role, because $\gamma^{(2)}$ and $Z^{(2)}$ can decay into dileptons through these couplings. Next we have shown all significant processes for $\gamma^{(2)}$ productions. Then we have calculated the production cross sections of $\gamma^{(2)}$ and $Z^{(2)}$. Finally we have found that the number of dilepton events are expected to be more than 1 for $1 / R \lesssim 1600 \mathrm{GeV}$ with an integrated luminosity of $100 \mathrm{fb}^{-1}$. We need further study to discuss the feasibility for the discovery of the MUED model by estimating the background from the SM. We leave it for a future work. ${ }^{10}$

\section{Acknowledgments}

The work of J. S. was supported in part by the Grant-in-Aid for the Ministry of Education, Culture, Sports, Science, and Technology, Government of Japan (No. 20025001, 20039001, and 20540251). The work of M. Y. was supported in part by the Grant-in-Aid for the Ministry of Education, Culture, Sports, Science, and Technology, Government of Japan (No. 20007555).

\section{References}

1. T. Appelquist, H. C. Cheng and B. A. Dobrescu, Phys. Rev. D 64 (2001) 035002.

2. H. C. Cheng, K. T. Matchev and M. Schmaltz, Phys. Rev. D 66 (2002) 036005.

3. H. C. Cheng, J. L. Feng and K. T. Matchev, Phys. Rev. Lett. 89 (2002) 211301; G. Servant and T. M. P. Tait, Nucl. Phys. B 650 (2003) 391.

4. S. Matsumoto, J. Sato, M. Senami and M. Yamanaka, Phys. Lett. B 647 (2007) 466.

5. M. Kakizaki, S. Matsumoto, Y. Sato and M. Senami, Phys. Rev. D 71 (2005) 123522; S. Matsumoto and M. Senami, Phys. Lett. B 633 (2006) 671; F. Burnell and G. D. Kribs, Phys. Rev. D 73 (2006) 015001; M. Kakizaki, S. Matsumoto and M. Senami, Phys. Rev. D 74 (2006) 023504; K. Kong and K. T. Matchev, JHEP 0601 (2006) 038; S. Matsumoto, J. Sato, M. Senami and M. Yamanaka, Phys. Rev. D 76 (2007) 043528 .

6. A. Datta, K. Kong and K. T. Matchev, Phys. Rev. D 72 (2005) 096006 [Erratum-ibid. D 72 (2005) 119901].

7. S. Matsumoto, J. Sato, M. Senami and M. Yamanaka, arXiv:0903.3255 [hep-ph].

8. J. Pumplin, D. R. Stump, J. Huston, H. L. Lai, P. Nadolsky and W. K. Tung, JHEP 0207 (2002) 012.

9. A. Pukhov, arXiv:hep-ph/0412191

10. S. Matsumoto, J. Sato, M. Senami and M. Yamanaka, work in progress. 